\title{
Feedback Numbers of Goldberg Snark, Twisted Goldberg Snarks and Related Graphs
}

\author{
Xinyue Zhang ${ }^{1}$, Yijin Wang ${ }^{1}$ and Sijia Zhang ${ }^{1, a}$ \\ ${ }^{1}$ School of Information Engineering, Dalian Ocean University, Dalian, 116023, China
}

\begin{abstract}
A subset of vertices of a graph $G$ is called a feedback vertex set of $G$ if its removal results in an acyclic subgraph. The minimum cardinality of a feedback vertex set is called the feedback number. In this paper, we determine the exact values of the feedback numbers of the Goldberg snarks $G_{n}$ and its related graphs $G_{n}{ }^{*}$, Twisted Goldberg Snarks $T G_{n}$ and its related graphs $T G_{n}{ }^{*}$. Let $f(n)$ denote the feedback numbers of these graphs, we prove that $f(n)=2 n+1$, for $n \geq 3$.
\end{abstract}

\section{Introduction}

Let $G=(V, E)$ be a graph or digraph without multiple edges, with vertex set $V(G)$ and edge set $E(G)$. A subset $F \subset V(G)$ is called a feedback vertex set if the subgraph $\mathrm{G}$ $-\mathrm{F}$ is acyclic, that is, if $G-F$ is a forest. The minimum cardinality of a feedback vertex set is called the feedback number (or decycling number proposed first by Beineke and Vandell [1]) of G. A feedback vertex set of this cardinality is called a minimum feedback vertex set.

Apart from its graph-theoretical interest, the minimum feedback vertex set problem has important application to several fields. For example, the problems are in operating systems to resource allocation mechanisms that prevent deadlocks [2], in artificial intelligence to the constraint satisfaction problem and Bayesian inference, in synchronous distributed systems to the study of monopolies and in optical networks to converters placement problem (see $[3,4])$.

Determining the feedback number is quite difficult even for some elementary graphs. However, the problem has been studied for some special graphs and digraphs, such as hypercubes, meshes, toroids, butterflies, cubeconnected cycles, directed split-stars (see $[3,4,5,6,7,8$, $9,10,11,12,13])$. In fact, the minimum feedback set problem is known to be NP-hard for general graphs [14] and the best known approximation algorithm is one with an approximation ratio two [5].

Snarks are simple nontrivial connectes cubic graphs of chromatic index four [16]. The importance of the snar-ks does not only depend on the four colour theorem. Indeed, there are several important open problems such as the classical cycle double cover conjecture [18]. The smallest snark is the Petersen graph and it was the first discovered. In [17], it gave a survey of some results on some well known families of snarks, e.g. Flower snarks, Loupekine snarks and Goldberg snarks.

Goldberg Snarks is referred to Loupekine by Goldberg himself. In fact, Goldberg Snarks are cartwheel snarks [19]. Both Goldberg Snarks and twisted Goldberg Snarks are graphs on $8 n$ vertices with

$$
V\left(G_{n}\right)=\left\{v_{j}^{i} \mid 0 \leq i \leq n-1,1 \leq j \leq 8\right\}
$$

- For every odd $n \geq 3, G_{n}$ is called the Goldberg Snark graph and adjacencies are defined as shown in Fig.1, where the vertex labels $i$ are read modulo $n$.

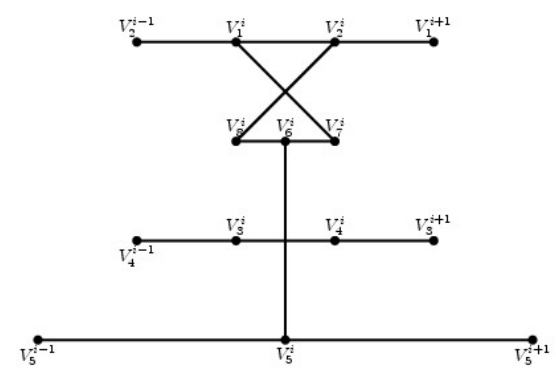

Figure 1. Used in the construction of Goldberg snark

- For every even $n \geq 4, G_{n}{ }^{*}$ is called Related graphs of Goldberg Snark.

- For every odd $n \geq 3$, let $T G_{n}$ be a graph obtained from $G_{n}$ by replacing the edges $v_{2}^{n-1} v_{1}^{0}$ and $v_{4}^{n-1} v_{3}^{0}$ with $v_{2}^{n-1} v_{3}^{0}$ and $v_{4}^{n-1} v_{1}^{0}$, respectively. We denote $T G_{n}$ as Twisted Goldberg Snark.

- For every even $n \geq 4, T G_{n}{ }^{*}$ is called Related graphs of Twisted Goldberg Snark.

a Corresponding author: zhangsijia@dlou.edu.cn 
Fig. 2 .

The example of $G_{3}, G_{4}{ }^{*}, T G_{3}$ and $T G_{4}{ }^{*}$ are shown in

However, both Goldberg Snarks and twisted Goldberg Snarks can be obtained via the Loupekine construction from the Petersen graph [15].

In this paper, we determine the exact values of the feedback number of the Goldberg snarks $G_{n}$ and its related graphs $G_{n}{ }^{*}$, the twisted Goldberg snarks $T G_{n}$ and its related graphs $T G_{n}{ }^{*}$. Let $f(n)$ denote the feedback number of these graphs, we prove that $f(n)=2 n+1$, for $n \geq 3$.
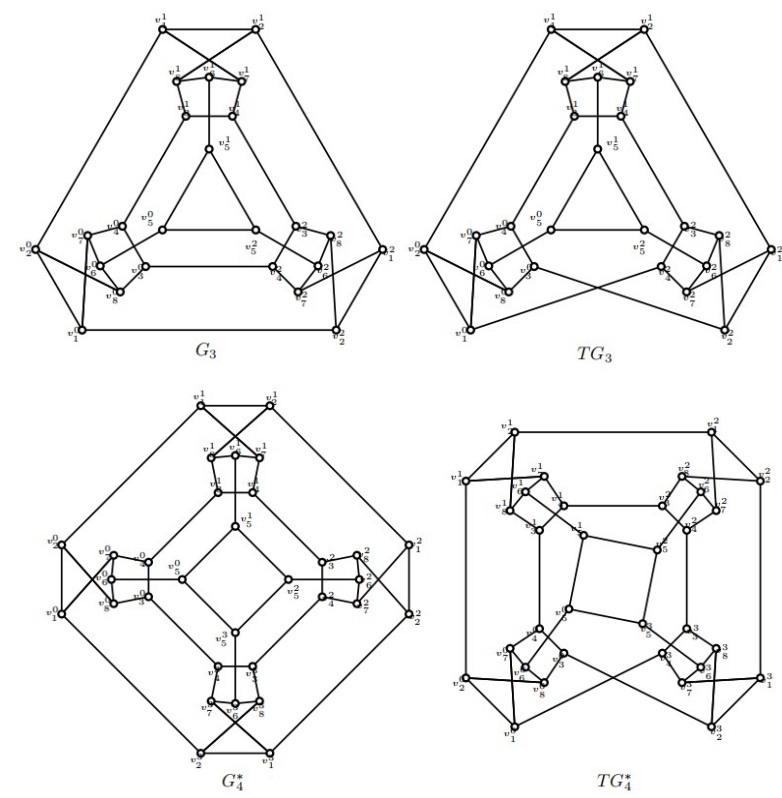

Figure 2. Graph $G_{3}, G_{4}{ }^{*}, T G_{3}$ and $T G_{4}{ }^{*}$

\section{Acyclic Vertex Set of $\boldsymbol{G}_{\boldsymbol{n}}$ and $\boldsymbol{G}_{\boldsymbol{n}}{ }^{*}$}

Let $n \bmod 2=k$, then $n=2 m+k, m \geq 1$ and $0 \leq i \leq k$. Thus $k=0$ and $k=1$. Then, we discuss 2 cases as follows.

Case 1. If $n \bmod 2=1$ and $n \geq 3$

Let $V_{a}\left(G_{n}\right)=F_{1}^{n} \cup F_{2}^{n}$, where

$$
\begin{aligned}
& F_{1}^{n}=\left\{v_{2}^{0}, v_{3}^{0}, v_{8}^{0}, v_{6}^{0}, v_{7}^{0}\right\} \\
& F_{2}^{n}=\bigcup_{i=1}^{n-1}\left\{v_{2}^{i}, v_{3}^{i}, v_{8}^{i}, v_{6}^{i}, v_{7}^{i}, v_{5}^{i}\right\}
\end{aligned}
$$

Case 2. If $n \bmod 2=0$ and $n \geq 4$.

Let $V_{a}\left(G_{n}^{*}\right)=F_{1}^{n} \cup F_{2}^{n} \cup F_{3}^{n}$, where

$$
\begin{aligned}
& F_{1}^{n}=\left\{v_{2}^{0}, v_{3}^{0}, v_{8}^{0}, v_{6}^{0}, v_{7}^{0}\right\} \\
& F_{2}^{n}=\left\{v_{2}^{1}, v_{3}^{1}, v_{8}^{1}, v_{6}^{1}, v_{7}^{1}, v_{5}^{1}\right\} \\
& F_{3}^{n}=\bigcup_{i=2}^{n-1}\left\{v_{2}^{i}, v_{3}^{i}, v_{8}^{i}, v_{6}^{i}, v_{7}^{i}, v_{5}^{i}\right\}
\end{aligned}
$$

Lemma 2.1. $G\left[V_{a}\left(G_{n}\right)\right]$ is acyclic for $n \bmod 2=1$ and $n \geq 3$.
Proof: Since $V_{a}\left(G_{n}\right)=F_{1}^{n} \cup F_{2}^{n}$, Then if we want to prove $G\left[V_{a}\left(G_{n}\right)\right]$ is acyclic, which is equivalent to verify that $G\left[F_{1}^{n} \cup F_{1}^{n}\right]$ is acyclic.

Firstly, we prove the $G\left[F_{1}^{n} \cup F_{1}^{n}\right]$ is a forest by induction on $n$.

We first prove the basic step for $n=3$.

Combining case 1 and the definition of $G_{n}$, we have

$$
\begin{aligned}
& F_{1}^{3}=\left\{v_{2}^{0}, v_{3}^{0}, v_{8}^{0}, v_{6}^{0}, v_{7}^{0}\right\} \\
& F_{2}^{3}=\left\{v_{2}^{1}, v_{3}^{1}, v_{8}^{1}, v_{6}^{1}, v_{7}^{1}, v_{5}^{1}, v_{2}^{2}, v_{3}^{2}, v_{8}^{2}, v_{6}^{2}, v_{7}^{2}, v_{5}^{2}\right\}
\end{aligned}
$$

Obviously, the induced subgraph of vertex set $F_{1}^{3}$ and the induced subgraph of vertex set $F_{2}^{3}$ are acyclic graphs(see Fig.3 and Fig.4) and each graph of $G\left[F_{1}^{3}\right]$ and $G\left[F_{2}^{3}\right]$ is a tree.

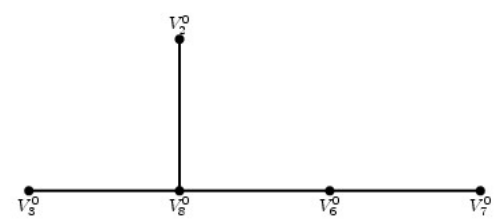

Figure 3. Subgraph of $G\left[F_{1}^{3}\right]$

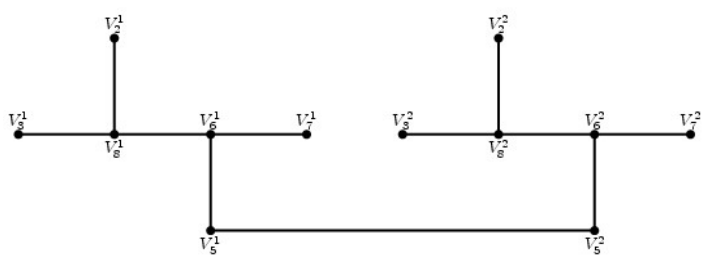

Figure 4. Subgraph of $G\left[F_{2}^{3}\right]$

Since $F_{1}^{3} \cap F_{2}^{3}=\varnothing$, then the induced subgraph of vertex set $F_{1}^{3} \cup F_{2}^{3}$ is acyclic. That is, $G\left[F_{1}^{3} \cup F_{2}^{3}\right]$ is a forest.

Suppose $G\left[F_{1}^{n} \cup F_{2}^{n}\right]$ is acyclic, we now prove that $G\left[F_{1}^{n+2} \cup F_{2}^{n+2}\right]$ is acyclic.

Since

$$
\begin{aligned}
& F_{1}^{n+2}=\left\{v_{2}^{0}, v_{3}^{0}, v_{8}^{0}, v_{6}^{0}, v_{7}^{0}\right\} \\
& F_{2}^{n+2}=\bigcup_{i=1}^{n+2-1}\left\{v_{2}^{i}, v_{3}^{i}, v_{8}^{i}, v_{6}^{i}, v_{7}^{i}, v_{5}^{i}\right\}
\end{aligned}
$$

Then we have 


$$
\begin{aligned}
F_{1}^{n+2}= & F_{1}^{n} \\
F_{2}^{n+2}= & \bigcup_{i=1}^{n-1}\left\{v_{2}^{i}, v_{3}^{i}, v_{8}^{i}, v_{6}^{i}, v_{7}^{i}, v_{5}^{i}\right\} \\
& \cup \bigcup_{i=n}^{n+1}\left\{v_{2}^{i}, v_{3}^{i}, v_{8}^{i}, v_{6}^{i}, v_{7}^{i}, v_{5}^{i}\right\} \\
= & F_{2}^{n} \cup \bigcup_{i=n}^{n+1}\left\{v_{2}^{i}, v_{3}^{i}, v_{8}^{i}, v_{6}^{i}, v_{7}^{i}, v_{5}^{i}\right\}
\end{aligned}
$$

For convenience, we denote $T$ as vertex set $\bigcup_{i=n}^{n+1}\left\{v_{2}^{i}, v_{3}^{i}, v_{8}^{i}, v_{6}^{i}, v_{7}^{i}, v_{5}^{i}\right\}$, then we denote the induced subgraph of vertex set $\bigcup_{i=n}^{n+1}\left\{v_{2}^{i}, v_{3}^{i}, v_{8}^{i}, v_{6}^{i}, v_{7}^{i}, v_{5}^{i}\right\}$ as $G[T]$.

Obviously, $G[T] \cong G\left[F_{2}^{3}\right]$ (see Fig. 5), then $G[T]$ is a tree.

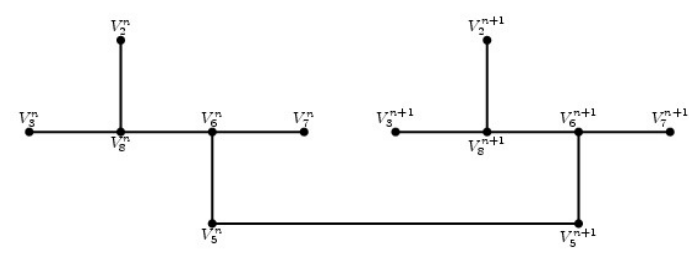

Figure 5. Subgraph of $G[T]$

Since $T \cap F_{2}^{n}=\varnothing$, then $G\left[T \cup F_{2}^{n}\right]$ is a forest.

Since $T \cap F_{1}^{n}=\varnothing$, then $G\left[T \cup F_{1}^{n} \cup F_{2}^{n}\right]$ is a forest. That is, $G\left[F_{1}^{n+2} \cup F_{2}^{n+2}\right]$ is acyclic.

Combining induction step with basic step, the $G\left[F_{1}^{n} \cup F_{2}^{n}\right]$ is a forest. Thus, $G\left[V_{a}\left(G_{n}\right)\right]$ is acyclic.

The lemma holds.

Lemma 2.2. $G\left[V_{a}\left(G_{n}^{*}\right)\right]$ is acyclic for $n \bmod 2=0$ and $n \geq 4$.

Proof: Since $V_{a}\left(G_{n}^{*}\right)=F_{1}^{n} \cup F_{2}^{n} \cup F_{3}^{n}$, Then if we want to prove $G\left[V_{a}\left(G_{n}^{*}\right)\right]$ is acyclic, which is equivalent to verify that $G\left[F_{1}^{n} \cup F_{2}^{n} \cup F_{3}^{n}\right]$ is acyclic.

Firstly, we prove the $G\left[F_{1}^{n} \cup F_{2}^{n} \cup F_{3}^{n}\right]$ is a forest by induction on $n$.

We first prove the basic step for $n=4$.

Combining case 2 and the definition of $G_{n}{ }^{*}$, we have

$$
\begin{aligned}
& F_{1}^{4}=\left\{v_{2}^{0}, v_{3}^{0}, v_{8}^{0}, v_{6}^{0}, v_{7}^{0}\right\} \\
& F_{2}^{4}=\left\{v_{2}^{1}, v_{3}^{1}, v_{8}^{1}, v_{6}^{1}, v_{7}^{1}, v_{5}^{1}\right\} \\
& F_{3}^{4}=\left\{v_{2}^{2}, v_{3}^{2}, v_{8}^{2}, v_{6}^{2}, v_{7}^{2}, v_{5}^{2}, v_{2}^{3}, v_{3}^{3}, v_{8}^{3}, v_{6}^{3}, v_{7}^{3}, v_{5}^{3}\right\}
\end{aligned}
$$

Obviously, $G\left[F_{1}^{4}\right], G\left[F_{2}^{4}\right]$ and $G\left[F_{3}^{4}\right]$ are acyclic graphs, and each graph of $G\left[F_{1}^{4}\right], G\left[F_{2}^{4}\right]$ and $G\left[F_{3}^{4}\right]$ is a tree.

Since $F_{1}^{4} \cap F_{2}^{4}=\varnothing$, then the induced subgraph of vertex set $F_{1}^{4} \cup F_{2}^{4}$ is acyclic.
Since $F_{1}^{4} \cap F_{3}^{4}=\varnothing$, then the induced subgraph of vertex set $F_{1}^{4} \cup F_{3}^{4}$ is acyclic.

Since $F_{2}^{4} \cap F_{3}^{4}=\varnothing$, then the induced subgraph of vertex set $F_{2}^{4} \cup F_{3}^{4}$ is acyclic.

Thus, we have $F_{1}^{4} \cap F_{2}^{4} \cap F_{3}^{4}=\varnothing$, then the induced subgraph of vertex set $F_{1}^{4} \cup F_{2}^{4} \cup F_{3}^{4}$ is acyclic.

That is, $G\left[F_{1}^{4} \cup F_{2}^{4} \cup F_{3}^{4}\right]$ is a forest. The vertices set is shown as Fig 6.

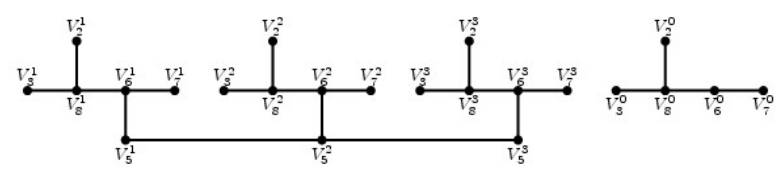

Figure 6. Subgraph of $G\left[F_{1}^{4} \cup F_{2}^{4} \cup F_{2}^{4}\right]$

Suppose $G\left[F_{1}^{n} \cup F_{2}^{n} \cup F_{3}^{n}\right]$ is a forest, we now prove that $G\left[F_{1}^{n+2} \cup F_{2}^{n+2} \cup F_{3}^{n+2}\right]$ is a forest.

Since

$$
\begin{aligned}
& F_{1}^{n+2}=\left\{v_{2}^{0}, v_{3}^{0}, v_{8}^{0}, v_{6}^{0}, v_{7}^{0}\right\} \\
& F_{2}^{n+2}=\left\{v_{2}^{1}, v_{3}^{1}, v_{8}^{1}, v_{6}^{1}, v_{7}^{1}, v_{5}^{1}\right\} \\
& F_{3}^{n+2}=\bigcup_{i=1}^{n+2-1}\left\{v_{2}^{i}, v_{3}^{i}, v_{8}^{i}, v_{6}^{i}, v_{7}^{i}, v_{5}^{i}\right\}
\end{aligned}
$$

Then, we have

$$
\begin{aligned}
F_{1}^{n+2}= & F_{1}^{n} \\
F_{2}^{n+2}= & F_{2}^{n} \\
F_{3}^{n+2}= & \bigcup_{i=2}^{n-1}\left\{v_{2}^{i}, v_{3}^{i}, v_{8}^{i}, v_{6}^{i}, v_{7}^{i}, v_{5}^{i}\right\} \\
& \cup \bigcup_{i=n}^{n+1}\left\{v_{2}^{i}, v_{3}^{i}, v_{8}^{i}, v_{6}^{i}, v_{7}^{i}, v_{5}^{i}\right\} \\
= & F_{3}^{n} \cup \bigcup_{i=n}^{n+1}\left\{v_{2}^{i}, v_{3}^{i}, v_{8}^{i}, v_{6}^{i}, v_{7}^{i}, v_{5}^{i}\right\}
\end{aligned}
$$

For convenience, we also denote $T$ as vertex set $\bigcup_{i=n}^{n+1}\left\{v_{2}^{i}, v_{3}^{i}, v_{8}^{i}, v_{6}^{i}, v_{7}^{i}, v_{5}^{i}\right\}$, then we denote the induced subgraph of vertex set $\bigcup_{i=n}^{n+1}\left\{v_{2}^{i}, v_{3}^{i}, v_{8}^{i}, v_{6}^{i}, v_{7}^{i}, v_{5}^{i}\right\}$ as $G[T]$.

Obviously, $G[T] \cong G\left[F_{3}^{4}\right]$, then $G[T]$ is a tree.

Since $T \cap F_{3}^{n}=\varnothing$, then $G\left[T \cup F_{3}^{n}\right]$ is a forest.

Since $T \cap F_{1}^{n}=\varnothing$, then $G\left[T \cup F_{1}^{n} \cup F_{2}^{n}\right]$ is a forest.

Since $T \cap F_{2}^{n}=\varnothing$, then $G\left[T \cup F_{1}^{n} \cup F_{2}^{n} \cup F_{3}^{n}\right]$ is a forest. That is, $G\left[F_{1}^{n+2} \cup F_{2}^{n+2} \cup F_{3}^{n+2}\right]$ is acyclic. 
Combining induction step with basic step, the $G\left[F_{1}^{n} \cup F_{2}^{n} \cup F_{3}^{n}\right]$ is a forest. Thus, $G\left[V_{a}\left(G_{n}^{*}\right)\right]$ is acyclic.

The lemma holds.

\section{Acyclic Vertex Set of $T G_{n}$ and $T G_{n}{ }^{*}$}

Let $n \bmod 2=k$, then $n=2 m+k, m \geq 1$ and $0 \leq i \leq k$. Thus $k=0$ and $k=1$. Then, we discuss 2 cases as follows.

Case 1. If $n \bmod 2=1$ and $n \geq 3$

Let $V_{a}\left(T G_{n}\right)=F_{1}^{n} \cup F_{2}^{n}$, where

$$
\begin{aligned}
& F_{1}^{n}=\left\{v_{2}^{0}, v_{3}^{0}, v_{8}^{0}, v_{6}^{0}, v_{7}^{0}, v_{2}^{n-1}, v_{3}^{n-1}, v_{8}^{n-1}, v_{6}^{n-1}, v_{7}^{n-1}, v_{5}^{n-1}\right\} \\
& F_{2}^{n}=\bigcup_{i=1}^{n-2}\left\{v_{2}^{i}, v_{3}^{i}, v_{8}^{i}, v_{6}^{i}, v_{7}^{i}, v_{5}^{i}\right\}
\end{aligned}
$$

Case 2. If $n \bmod 2=0$, and $n \geq 4$

Let $V_{a}\left(T G_{n}^{*}\right)=F_{1}^{n} \cup F_{2}^{n}$, where

$$
\begin{aligned}
& F_{1}^{n}=\left\{v_{2}^{0}, v_{3}^{0}, v_{8}^{0}, v_{6}^{0}, v_{7}^{0}, v_{2}^{n-1}, v_{3}^{n-1}, v_{8}^{n-1}, v_{6}^{n-1}, v_{7}^{n-1}, v_{5}^{n-1}\right\} \\
& F_{2}^{n}=\bigcup_{i=1}^{n-2}\left\{v_{2}^{i}, v_{3}^{i}, v_{8}^{i}, v_{6}^{i}, v_{7}^{i}, v_{5}^{i}\right\}
\end{aligned}
$$

Lemma 3.1. $G\left[V_{a}\left(T G_{n}\right)\right]$ is acyclic for $n \bmod 2=1$ and $n \geq 3$.

Proof: Since $V_{a}\left(T G_{n}\right)=F_{1}^{n} \cup F_{2}^{n}$, Then if we want to prove $G\left[V_{a}\left(T G_{n}\right)\right]$ is acyclic, which is equivalent to verify that $G\left[F_{1}^{n} \cup F_{2}^{n}\right]$ is acyclic.

Firstly, we prove the $G\left[F_{1}^{n} \cup F_{2}^{n}\right]$ is a tree by induction on $n$.

We first prove the basic step for $n=3$.

Combining case 1 and the definition of $T G_{n}$, we have

$$
\begin{aligned}
& F_{1}^{3}=\left\{v_{2}^{0}, v_{3}^{0}, v_{8}^{0}, v_{6}^{0}, v_{7}^{0}, v_{2}^{2}, v_{3}^{2}, v_{8}^{2}, v_{6}^{2}, v_{7}^{2}, v_{5}^{2}\right\} \\
& F_{2}^{3}=\left\{v_{2}^{1}, v_{3}^{1}, v_{8}^{1}, v_{6}^{1}, v_{7}^{1}, v_{5}^{1}\right\}
\end{aligned}
$$

Obviously, by the definition of $T \mathrm{G}_{n}$, we have that $G\left[F_{1}^{3}\right]$ and $G\left[F_{2}^{3}\right]$ are acyclic graphs, and each graph of $G\left[F_{1}^{3}\right]$ and $G\left[F_{2}^{3}\right]$ is a tree.

Since $F_{1}^{3} \cap F_{2}^{3}=\varnothing$, then the induced subgraph of vertex set $F_{1}^{3} \cup F_{2}^{3}$ is acyclic. That is, $G\left[F_{1}^{3} \cup F_{2}^{3}\right]$ is a tree. The vertices set is shown as Fig 7.
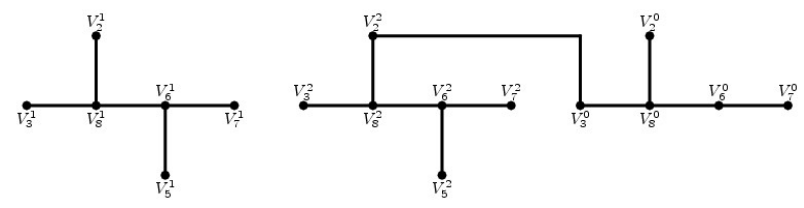

Figure 7. Subgraph of $G\left[F_{1}^{3} \cup F_{2}^{3}\right]$

Suppose $G\left[F_{1}^{n} \cup F_{2}^{n}\right]$ is a tree, we now prove that $G\left[F_{1}^{n+2} \cup F_{2}^{n+2}\right]$ is a tree.
Since

$$
\begin{aligned}
F_{1}^{n+2} & =\left\{v_{2}^{0}, v_{3}^{0}, v_{8}^{0}, v_{6}^{0}, v_{7}^{0}, v_{2}^{n+1}, v_{3}^{n+1}, v_{8}^{n+1}, v_{6}^{n+1}, v_{7}^{n+1}, v_{5}^{n+1}\right\} \\
F_{2}^{n+2} & =\bigcup_{i=1}^{n+2-2}\left\{v_{2}^{i}, v_{3}^{i}, v_{8}^{i}, v_{6}^{i}, v_{7}^{i}, v_{5}^{i}\right\} \\
& =\bigcup_{i=1}^{n-2}\left\{v_{2}^{i}, v_{3}^{i}, v_{8}^{i}, v_{6}^{i}, v_{7}^{i}, v_{5}^{i}\right\} \cup \bigcup_{i=n-1}^{n}\left\{v_{2}^{i}, v_{3}^{i}, v_{8}^{i}, v_{6}^{i}, v_{7}^{i}, v_{5}^{i}\right\} \\
& =F_{2}^{n} \cup \bigcup_{i=n-1}^{n}\left\{v_{2}^{i}, v_{3}^{i}, v_{8}^{i}, v_{6}^{i}, v_{7}^{i}, v_{5}^{i}\right\}
\end{aligned}
$$

Then we have

$$
\begin{aligned}
& F_{1}^{n+2} \cong F_{1}^{n} \\
& F_{2}^{n+2}=F_{2}^{n} \cup \bigcup_{i=n-1}^{n}\left\{v_{2}^{i}, v_{3}^{i}, v_{8}^{i}, v_{6}^{i}, v_{7}^{i}, v_{5}^{i}\right\}
\end{aligned}
$$

For convenience, we denote $T$ as vertex set $\bigcup_{i=n-1}^{n}\left\{v_{2}^{i}, v_{3}^{i}, v_{8}^{i}, v_{6}^{i}, v_{7}^{i}, v_{5}^{i}\right\}$, then we denote the induced subgraph of vertex set $\bigcup_{i=n-1}^{n}\left\{v_{2}^{i}, v_{3}^{i}, v_{8}^{i}, v_{6}^{i}, v_{7}^{i}, v_{5}^{i}\right\}$ as $G[T]$.

Obviously, $G[T] \cong G\left[F_{2}^{3}\right]$, then $G[T]$ is a tree.

Since $T \cap F_{2}^{n}=\varnothing$, then $G\left[T \cup F_{2}^{n}\right]$ is a tree.

Since $T \cap F_{1}^{n}=\varnothing$, then $G\left[T \cup F_{1}^{n} \cup F_{2}^{n}\right]$ is a tree. That is, $G\left[F_{1}^{n+2} \cup F_{2}^{n+2}\right]$ is acyclic.

Combining induction step with basic step, the $G\left[F_{1}^{n} \cup F_{2}^{n}\right]$ is a tree. Thus, $G\left[V_{a}\left(T G_{n}\right)\right]$ is acyclic.

The lemma holds.

Lemma 3.2. $G\left[V_{a}\left(T G_{n}^{*}\right)\right]$ is acyclic for $n \bmod 2=0$ and $n \geq 4$.

Proof: Since $V_{a}\left(T G_{n}^{*}\right)=F_{1}^{n} \cup F_{2}^{n}$, Then if we want to prove $G\left[V_{a}\left(T G_{n}^{*}\right)\right]$ is acyclic, which is equivalent to verify that $G\left[F_{1}^{n} \cup F_{2}^{n}\right]$ is acyclic.

Firstly, we prove the $G\left[F_{1}^{n} \cup F_{2}^{n}\right]$ is a tree by induction on $n$.

We first prove the basic step for $n=4$.

Combining case 2 and the definition of $T G_{n}^{*}$,we have

$$
\begin{aligned}
& F_{1}^{4}=\left\{v_{2}^{0}, v_{3}^{0}, v_{8}^{0}, v_{6}^{0}, v_{7}^{0}, v_{2}^{3}, v_{3}^{3}, v_{8}^{3}, v_{6}^{3}, v_{7}^{3}, v_{5}^{3}\right\} \\
& F_{2}^{4}=\left\{v_{2}^{1}, v_{3}^{1}, v_{8}^{1}, v_{6}^{1}, v_{7}^{1}, v_{5}^{1}, v_{2}^{2}, v_{3}^{2}, v_{8}^{2}, v_{6}^{2}, v_{7}^{2}, v_{5}^{2}\right\}
\end{aligned}
$$

Obviously, $G\left[F_{1}^{4}\right]$ and $G\left[F_{2}^{4}\right]$ are acyclic graph, and each graph of $G\left[F_{1}^{4}\right]$ and $G\left[F_{2}^{4}\right]$ is a tree. Since $F_{1}^{4} \cap F_{2}^{4}=\varnothing$, then the induced subgraph of vertex set $F_{1}^{4} \cup F_{2}^{4}$ is acyclic. That is, $G\left[F_{1}^{4} \cup F_{2}^{4}\right]$ is a tree. The vertices set is shown as Fig 8. 


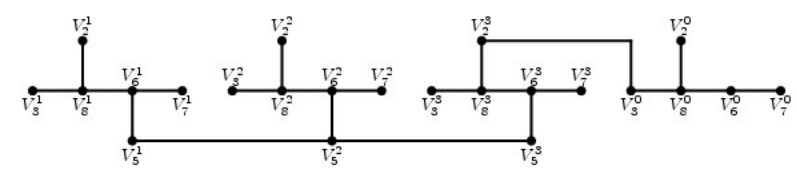

Figure 8. Subgraph of $G\left[F_{1}^{4} \cup F_{2}^{4}\right]$

Suppose $G\left[F_{1}^{n} \cup F_{2}^{n}\right]$ is a tree, we now prove that $G\left[F_{1}^{n+2} \cup F_{2}^{n+2}\right]$ is a tree.

Since

$$
\begin{aligned}
F_{1}^{n+2}= & \left\{v_{2}^{0}, v_{3}^{0}, v_{8}^{0}, v_{6}^{0}, v_{7}^{0}, v_{2}^{n-1}\right\} \\
& \cup\left\{v_{3}^{n+1}, v_{8}^{n+1}, v_{6}^{n+1}, v_{7}^{n+1}, v_{5}^{n+1}\right\} \\
F_{2}^{n+2}= & \bigcup_{i=1}^{n+2-2}\left\{v_{2}^{i}, v_{3}^{i}, v_{8}^{i}, v_{6}^{i}, v_{7}^{i}, v_{5}^{i}\right\}
\end{aligned}
$$

Then we have

$$
\begin{aligned}
& F_{1}^{n+2} \cong F_{1}^{n} \\
& F_{2}^{n+2}=F_{2}^{n} \cup \bigcup_{i=n-1}^{n}\left\{v_{2}^{i}, v_{3}^{i}, v_{8}^{i}, v_{6}^{i}, v_{7}^{i}, v_{5}^{i}\right\}
\end{aligned}
$$

For convenience, we also denote $T$ as vertex set $\bigcup_{i=n-1}^{n}\left\{v_{2}^{i}, v_{3}^{i}, v_{8}^{i}, v_{6}^{i}, v_{7}^{i}, v_{5}^{i}\right\}$, then we denote the induced subgraph of vertex set $\bigcup_{i=n-1}^{n}\left\{v_{2}^{i}, v_{3}^{i}, v_{8}^{i}, v_{6}^{i}, v_{7}^{i}, v_{5}^{i}\right\}$ as $G[T]$.

Obviously, $G[T] \cong G\left[F_{2}^{4}\right]$, then $G[T]$ is a tree.

Since $T \cap F_{2}^{n}=\varnothing$, then $G\left[T \cup F_{2}^{n}\right]$ is a tree.

Since $T \cap F_{1}^{n}=\varnothing$, then $G\left[T \cup F_{1}^{n} \cup F_{2}^{n}\right]$ is a tree. That is, $G\left[F_{1}^{n+2} \cup F_{2}^{n+2}\right]$ is acyclic.

Combining induction step with basic step, the $G\left[F_{1}^{n} \cup F_{2}^{n}\right]$ is a tree. Thus, $G\left[V_{a}\left(T G_{n}^{*}\right)\right]$ is acyclic.

The lemma holds.

\section{Feedback Number of graphs}

By the Lemma 2.1 and Lemma 2.2, we obtain

Lemma 4.1. $V\left(G_{n}\right) \backslash V_{a}\left(G_{n}\right)$ is feedback vertex set of $G_{n}$.

Lemma 4.2. $V\left(G_{n}^{*}\right) \backslash V_{a}\left(G_{n}^{*}\right)$ is feedback vertex set of $G_{n}^{*}$.

By the Lemma 3.1 and Lemma 3.2, we obtain

Lemma 4.3. $V\left(T G_{n}\right) \backslash V_{a}\left(T G_{n}\right)$ is feedback vertex set of $T G_{n}$.

Lemma 4.4. $V\left(T G_{n}^{*}\right) \backslash V_{a}\left(T G_{n}^{*}\right)$ is feedback vertex set of $T G_{n}^{*}$.
For convenience, we denote $f(n)$ as the feedback numbers of Goldberg Snark, Twist Goldberg Snark and their related graphs.

Lemma 4.5. For $n \geq 3$, the upper bound of feedback number is

$$
f(n) \leq 2 n+1
$$

Proof: By Lemma 4.1, we have the feedback number of $V\left(G_{n}\right)$ as follows.

$$
\begin{aligned}
& \left|V\left(G_{n}\right) \backslash V_{a}\left(G_{n}\right)\right| \\
\leq & \left|V\left(G_{n}\right) \backslash\left(F_{1}^{n} \cup F_{2}^{n}\right)\right| \\
= & \left|V\left(G_{n}\right)\right|-\left|\left(F_{1}^{n} \cup F_{2}^{n}\right)\right| \\
= & 8 n-[5+6(n-1)] \\
= & 2 n+1
\end{aligned}
$$

By Lemma 4.2, we have the feedback number of $V\left(G_{n}^{*}\right)$ as follows.

$$
\begin{aligned}
& \left|V\left(G_{n}^{*}\right) \backslash V_{a}\left(G_{n}^{*}\right)\right| \\
\leq & \left|V\left(G_{n}^{*}\right) \backslash\left(F_{1}^{n} \cup F_{2}^{n} \cup F_{3}^{n}\right)\right| \\
= & \left|V\left(G_{n}^{*}\right)\right|-\left|\left(F_{1}^{n} \cup F_{2}^{n} \cup F_{3}^{n}\right)\right| \\
= & 8 n-[5+6+6(n-2)] \\
= & 2 n+1
\end{aligned}
$$

By Lemma 4.3, we have the feedback number of $V\left(T G_{n}\right)$ as follows.

$$
\begin{aligned}
& \left|V\left(T G_{n}\right) \backslash V_{a}\left(T G_{n}\right)\right| \\
\leq & \left|V\left(T G_{n}\right) \backslash\left(F_{1}^{n} \cup F_{2}^{n}\right)\right| \\
= & \left|V\left(T G_{n}\right)\right|-\left|\left(F_{1}^{n} \cup F_{2}^{n}\right)\right| \\
= & 8 n-[11+6(n-2)] \\
= & 2 n+1
\end{aligned}
$$

By Lemma 4.4, we have the feedback number of $V\left(T G_{n}\right)$ as follows.

$$
\begin{aligned}
& \left|V\left(T G_{n}^{*}\right) \backslash V_{a}\left(T G_{n}^{*}\right)\right| \\
\leq & \left|V\left(T G_{n}^{*}\right) \backslash\left(F_{1}^{n} \cup F_{2}^{n}\right)\right| \\
= & \left|V\left(T G_{n}^{*}\right)\right|-\left|\left(F_{1}^{n} \cup F_{2}^{n}\right)\right| \\
= & 8 n-[11+6(n-2)] \\
= & 2 n+1
\end{aligned}
$$

Then, we have the upper bound of feedback number of is $f(n) \leq 2 n+1$

Lemma 4.6. For $n \geq 3$, the lower bound of feedback number is

$$
f(n) \geq 2 n+1
$$

Proof: By reference [20], Beineke and Vandell prove a lower bound of general graphs $G(V, E)$ : 


$$
f(G) \geq\left\lceil\frac{|E|-|V|+1}{\Delta-1}\right\rceil
$$

Since $|V|=8 n,|E|=12 n$ and $\Delta=3$. Thus, we obtain a lower bound of these graphs as follows:

$$
\begin{aligned}
f(n) & \geq\left\lceil\frac{|E|-|V|+1}{\Delta-1}\right\rceil=\left\lceil\frac{12 n-8 n+1}{3-1}\right\rceil \\
& =\left\lceil\frac{4 n+1}{2}\right\rceil=\lceil 2 n+1\rceil
\end{aligned}
$$

By the Lemma 4.5 and Lemma 4.6, we obtain the feedback number of these graphs as follows.

Theorem1. For $n \geq 3$, the feedback number of these graphs is $f(n)=2 n+1$.

\section{Acknowledgment}

The work was supported by NNSF of China (No.61802046) and Key Research Items in Natural Science Foundation of Liaoning (No.20170520327) and Doctoral Start up Fund of Dalian Ocean University (No.HDYJ201818).

\section{References}

1. L. W. Beineke, R. C. Vandell, Decycling graphs, J. Graph Theory, 25, 59-77, (1997).

2. I. Niven, H. S. Zuckerman, An Introduction to the Theory of Numbers (5th ed.).John Wiley and Sons, New York, (1991).

3. I. Caragiannis, C. Kaklamanis, P. Kanellopoulos, New bounds on the size of the minimum feedback vertex set in meshes and butterflies. Information Processing Letters, 83, 75-80, (2002).

4. P. Festa, P. M. Pardalos, M. G. C. Resende, Feedback set problems. Handbook of Combinatorial Optimization (D.-Z. Du, P.M. Pardalos eds.), Vol. A, Kluwer, Dordrecht, pp. 209, (1999).

5. V. Bafna, P. Berman, T. Fujito, A 2-approximation algorithm for the undirected feedback vertex set problem. SIAM J. Discrete Mathematics, 12, 289297, (1999).

6. S. Bau, L. W. Beineke, Z. Liu, G. Du, R. C. Vandell, Decycling cubes and grids. Utilitas Math., 59, 129137, (2001).

7. R. Bar-Yehuda, D. Geiger, J. S. Naor, R. M. Roth, Approximation algorithms for the feedback vertex set problem with applications to constraint satisfaction and Bayesian inference. SIAM J. Comput., 27, 942959, (1998).
8. R. Focardi, F. L. Luccio, D. Peleg, Feedback vertex set in hypercubes. Information Processing Letters, 76, $1-5,(2000)$.

9. Y. D. Liang, On the feedback vertex set in permutation graphs. Information Pro cessing Letters, 52, 123-129, (1994).

10. F. L. Luccio, Almost exact minimum feedback vertex set in meshes and butterflies. Information Processing Letters, 66, 59-64, (1998).

11. G. W. Smith, Jr. and R. B. Walford, The identification of a minimal feedback vertex set of a directed graph. IEEE Trans. Circuits and Systems, 22, 9-15, (1975).

12. C.-C. Wang, E. L. Lloyd, M. L. Soffa, Feedback vertex sets and cyclically reducible graphs. J. Assoc. Comput. Mach., 32, 296-313, (1985).

13. F.-H. Wang, C.-J. Hsu, J.-C. Tsai, Minimal feedback vertex sets in directed split stars. Networks, 45, 218-223, (2005).

14. M.R. Garey, D.S. Johnson, Computers and Intractability, Freeman, San Francisco, CA, (1979).

15. Mohammad Ghebleh, The circular chromatic index of Goldberg snarks. Discrete Mathematics, 307, 3220-3225, (2007).

16. A.Cavicchioli, T.E. Murgolo, B. Ruini and F. Spaggiari. Special Classes of Snarks. Acta Applicandae Mathematicae, 76, 57-88, (2003).

17. A. Cavicchioli, M. Meschiari, B. Ruini, and F. Spaggiari. A Survey on Snarks and New Results: Products, Reducibility and a Computer Search. Journal of Graph Theory, 28(2), 57-86, (1998).

18. M. Abreu, D. Labbate, R. Rizzi, J. Sheehan, Odd 2factored snarks. European Journal of Combinatorics, 36, 460-472, (2014).

19. John J. Watrins, Snarks. Annals New York Academy of Sciences, 576, 606-622, (2006).

20. L. W. Beineke, R. C. Vandell, Decycling graphs, J. Graph Theory, 25, 59-77, (1997).

21. S.J. Zhang, X.R. Xu, C. Yin, N.Cao, Y.S. Yang, Feedback Numbers of Augmented Cubes AQn, Utilitas Mathematica, 97, 183-192, (2015)

22. S.J. Zhang, X.R. Xu, C. Yin, et al. The feedback number of Knödel graph W3, n, ARS Combinatoria, 140, 397-409, (2018).

23. X.R.Xu,C.Yin,S.J.Zhang, et al.Improved Feedback Vertex sets in Kautz Digraph Kautz Digraph K(d, n), CIS 2014, 161-165, (2014).

24. X.R. Xu, S.P. Dino, H.F. Zhang, et al.D ecycling number of crossed cubes CQn, CIS 2017, 145-150, (2017)

25. Forbes A.D.. Snark Design. Utilitas Mathematica, 107,167-192, (2018). 